\title{
Sensory recovery after infraorbital nerve avulsion injury
}

\author{
Sam Yong Lee, \\ Seung Hyun Kim, \\ Jae Ha Hwang, \\ Kwang Seog Kim \\ Department of Plastic and \\ Reconstructive Surgery, Chonnam \\ National University Medical School, \\ Gwangju, Korea
}

The infraorbital nerve is a branch of the trigeminal nerve. Injury to the infraorbital nerve can be caused by trauma, including various facial fractures. Due to this nerve injury, patients complain of numbness and pain in the entire cheek, the ala of nose, and upper lip. In general, spontaneous sensory recovery is expected after decompressive surgery. If nerve transection is confirmed, however, neurorrhaphy is typically performed. Here, we present a case in which microsurgery was not performed in a patient with Sunderland grade $V$ avulsion injury of the infraorbital nerve due to a facial bone fracture. Gradual nerve function recovery was confirmed to be possible with conservative treatment and rehabilitation alone. These findings suggest that the nerve function recovery can be expected with conservative treatment, even for severe nerve injury for which microsurgery cannot be considered.

Keywords: Evoked potentials / Nerve regeneration / Trigeminal nerve injuries

\section{INTRODUCTION}

The infraorbital nerve originates from the maxillary nerve, a branch of the trigeminal nerve, that runs along the inferior orbital fissure to reach the orbital cavity, and then passes through the infraorbital canal to come out of the infraorbital foramen and reaches the face [1]. It is responsible for the sensory innervation of the entire cheek, the ala of nose, and upper lip.

A peripheral nerve is a nerve that connects the central nervous system, including the brain and spinal cord, to the rest of the body, is fragile, and can be damaged easily. Nerve injury is divided into five stages in Sunderland's classification ranging from mild injuries, such as neuropraxia caused by compression, to the most severe form including total continuity loss caused by transection and avulsion injury [2]. The severer the nerve injury, the smaller the chance for regeneration and the longer time is needed to recover.

\section{Correspondence: Jae Ha Hwang}

Department of Plastic and Reconstructive Surgery, Chonnam National University

Medical School, 42 Jebong-ro, Dong-gu, Gwangju 61469, Korea

E-mail: psjhhwang@daum.net

Received July 6, 2020 / Revised July 27, 2020 / Accepted August 14, 2020
This case report observed a patient for 18 months and confirmed nerve function recovery following peripheral nerve regeneration in a patient with avulsion injury to the infraorbital nerve.

\section{CASE REPORT}

A 63-year-old man slipped and fell in a construction site and presented to the hospital with bilateral cheek swelling, left cheek numbness, and laceration wounds (Fig. 1). The patient complained of numbness of the lid cheek, nasolabial cheek, malar region, nasal alar region and upper lip without neuropathic pain or tingling sensation.

Facial computed tomography (CT) revealed Le Fort I fracture, left zygomaticomaxillary complex fracture, nasal bone fracture, and left orbital floor fracture (Fig. 2).

Upon physical examination an open fracture of the left maxillary anterior wall with comminution was seen.

During elective surgery in general anesthesia, open reduction and internal fixation were performed using absorbable plates and screws. Nevertheless, avulsion injury to the left infraorbital 

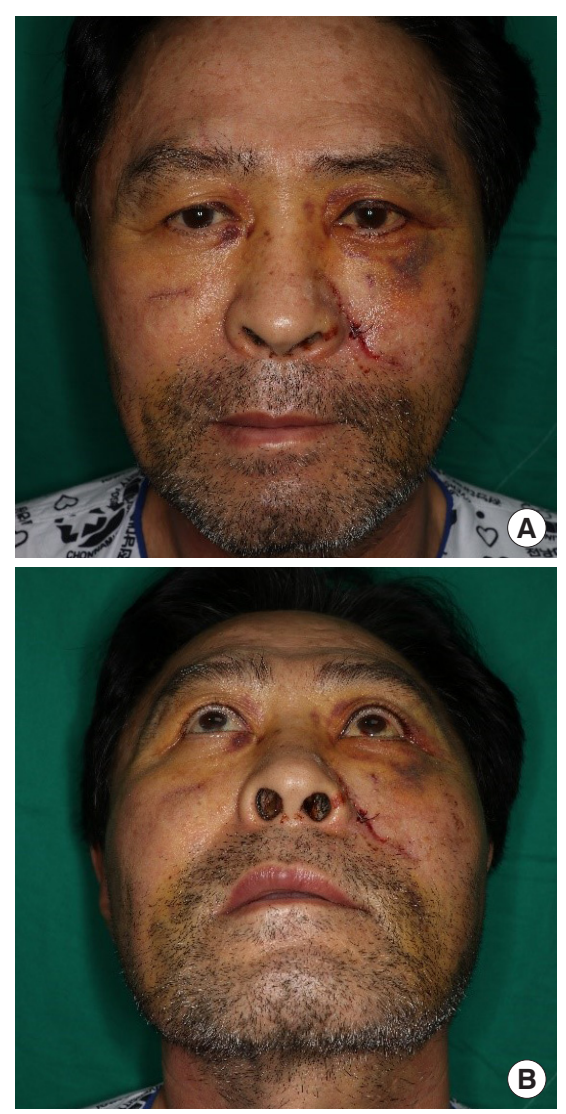

Fig. 1. The anteroposterior view (A) and the worm's-eye view (B) of the preoperative photographs. The complaints of the patient's first visit were bilateral cheek swelling, left cheek numbness and open wounds. The preoperative photos were taken when swelling decreased.

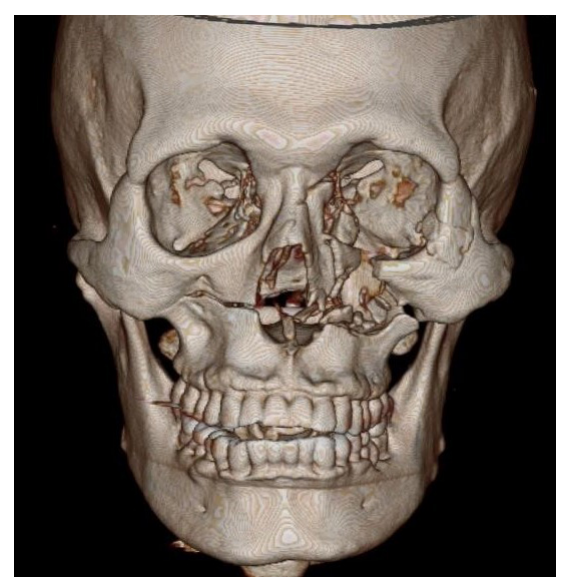

Fig. 2. The preoperative three-dimensional computed tomography (CT) imaging. The preoperative CT imaging shows left zygomaticomaxillary complex fracture, left orbital floor fracture, right maxilla fracture, and nasal bone fracture.

nerve was confirmed from the operative findings (Fig. 3). Microsurgical repair was not performed. The identified proximal portion of the distal nerve ending was located toward the infra-

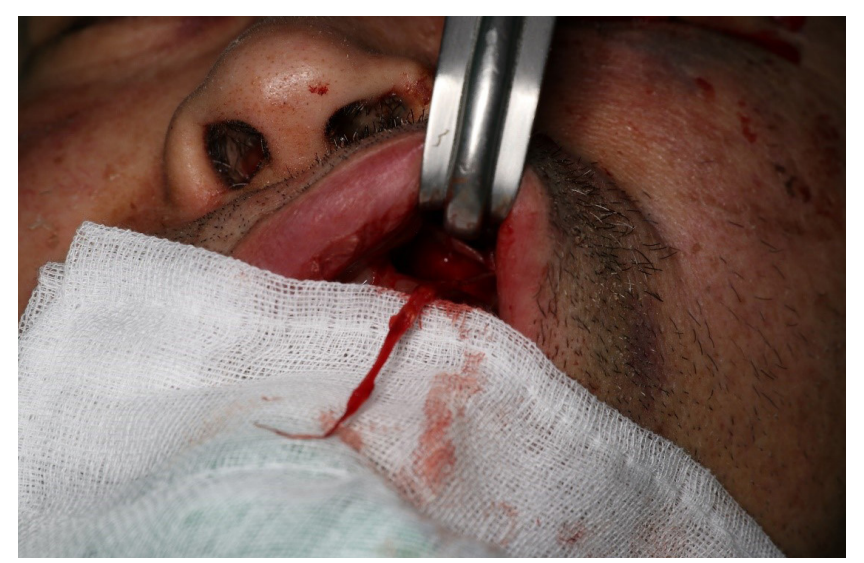

Fig. 3. The avulsion injury of inferior orbital nerve was confirmed from the intraoperative finding.

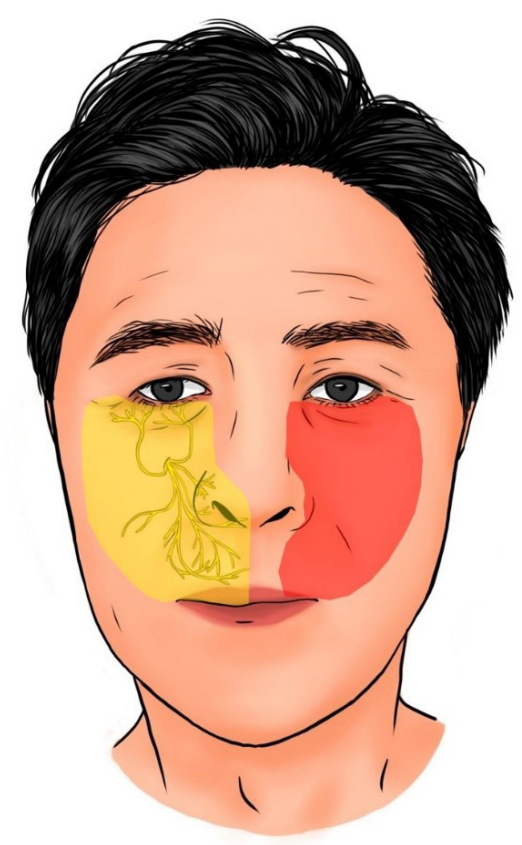

Fig. 4. Recovery pattern of the infraorbital nerve sensory dermatome. Sensation limited to the zygomatico-malar region at the time of the trauma was extended to the almost normal infraorbital nerve sensory dermatome. The right side of the face shows normal sensory dermatome (yellow-colored). The red-colored region represents the currently recovered infraorbital sensory dermatome except the nasal alar region and upper lip.

orbital foramen, and the operation was completed after incision closure.

Afterwards, the author consulted with the department of rehabilitation and, used electrical stimulation and infrared therapy to continue the patient's rehabilitation. The patient was followed with electrodiagnostic testing (nerve conduction study, blink reflex, and needle electromyography) every 6 months. The electrodiagnostic testing results confirmed injury to the left 
Table 1. The electrodiagnostic testing results

\begin{tabular}{|c|c|c|c|c|}
\hline \multirow{2}{*}{ Variable } & & \multicolumn{3}{|c|}{ Electrodiagnostic testing } \\
\hline & & 6 Months & 12 Months & 18 Months \\
\hline \multicolumn{5}{|c|}{ Sensory nerve conduction study } \\
\hline \multirow[t]{2}{*}{ Right } & Latency (ms) & 2.4 & 2.4 & 2.8 \\
\hline & Amplitude (mV) & 20.9 & 27.3 & 34.6 \\
\hline \multirow[t]{3}{*}{ Left } & Latency (ms) & 2.7 & 2.5 & 3.0 \\
\hline & Amplitude (mV) & 3.4 & 10.2 & 18.0 \\
\hline & Recovery rate (\%) & 16.3 & 37.4 & 52.0 \\
\hline \multicolumn{5}{|l|}{ Infraorbital blink reflex } \\
\hline \multirow[t]{2}{*}{ R1 Ipsilateral } & Right (ms) & 3.0 & 4.7 & 4.4 \\
\hline & Left (ms) & Not evoked & 6.7 & 5.1 \\
\hline \multirow[t]{2}{*}{ R2 Ipsilateral } & Right (ms) & 23.4 & 18.3 & 13.5 \\
\hline & Left (ms) & Not evoked & 28.9 & 14.2 \\
\hline \multirow[t]{2}{*}{ R2 Contralateral } & Right (ms) & 36.5 & 28.8 & 39.2 \\
\hline & Left (ms) & Not evoked & 30.4 & 35.7 \\
\hline \multicolumn{5}{|l|}{ Electromyography } \\
\hline \multirow[t]{2}{*}{ Left frontalis } & Spontaneous activity & Normal & Normal & Normal \\
\hline & MUAPS & Normal, discrete & Normal, discrete & Normal, discrete \\
\hline \multirow[t]{2}{*}{ Left nasalis } & Spontaneous activity & Positive sharp wave & Positive sharp wave, fibrillation & Normal \\
\hline & MUAPS & Normal, discrete & Polyphasic, discrete & Normal, discrete \\
\hline \multirow[t]{2}{*}{ Left orbicularis oris } & Spontaneous activity & Normal & Normal & Normal \\
\hline & MUAPS & Polyphasic, discrete & Normal, discrete & Normal, discrete \\
\hline \multicolumn{5}{|c|}{ Motor nerve conduction study } \\
\hline \multirow[t]{2}{*}{ Right nasalis } & Latency (ms) & 2.6 & 2.6 & 2.5 \\
\hline & Amplitude (mV) & 3.2 & 5.5 & 5.5 \\
\hline \multirow[t]{3}{*}{ Left nasalis } & Latency (ms) & 2.8 & 3.1 & 2.6 \\
\hline & Amplitude (mV) & 1.1 & 2.6 & 3.5 \\
\hline & Recovery rate (\%) & 34.3 & 47.2 & 63.3 \\
\hline
\end{tabular}

Axonal degeneration in the left trigeminal nerve was diagnosed by a decrease in amplitude in the sensory nerve conduction study, lesions in the infraorbital nerve and left facial nerve were diagnosed by left ipsilateral R1, R2 and contralateral R2 and right contralateral R2 delays observed in the infraorbital blink reflex test, and neuropathy of the buccal branch of the left facial nerve was diagnosed by discrete polyphasic motor unit action potentials (MUAPs) and abnormal spontaneous activity in the left nasalis and orbicularis oris observed using electromyography. Additionally, gradual nerve function recovery is demonstrated by improvements in these test results. The nerve conduction study showed a $52.0 \%$ recovery of infraorbital neuropathy and $63.6 \%$ recovery of facial neuropathy in 18 months.

infraorbital nerve and also to the buccal branch of the left facial nerve., The infraorbital nerve's function gradually improved from $16.3 \%$ at 6 months, to $37.4 \%$ at 12 months, and $52.0 \%$ at 18 months in a sensory nerve conduction study. The results of a blink reflex test also showed recovery of infraorbital nerve function (Table 1). A dull tactile sensation and recovery of nociception and thermoanesthesia were found in the previously numb areas (Fig. 4). The patient still showed incomplete left infraorbital neuropathy and left facial neuropathy. However, gradual recovery was observed in the patient's subjective symptoms.

\section{DISCUSSION}

One of the most common causes of injury to trigeminal nerve branches, including the infraorbital nerve, is accidental or iatro- genic trauma. Approximately $30 \%$ to $80 \%$ of patients with midfacial fractures experience infraorbital nerve injuries [3].

Infraorbital nerve injury causes symptoms like mild numbness in the sensory dermatome and sensory loss, ranging from complete anesthesia to dysesthesia $[4,5]$.

The severity of these symptoms is proportional to the severity of the nerve injury, which can be divided following Sunderland's classification defining grade I as neuropraxia caused by direct pressure or compression; grades II, III, and IV as more severe axonotmesis, each classified by a loss of axon continuity and demyelination, damage to the endoneurium, and damage to the perineurium caused by crushing or stretching; and grade $\mathrm{V}$ as neurotmesis, meaning a total loss of nerve trunk continuity caused by transection, laceration, and/or avulsion [2] .

The peripheral nerve is regenerated by the injured axon and 
non-neuronal cells, including Schwann cells, endoneurial fibroblasts, and macrophages. The proximal axon can regrow if the cell body is intact. The human axon's growth rate is $2 \mathrm{~mm} /$ day for small nerves and $5 \mathrm{~mm}$ /day for large nerves [6]. Furthermore, depending on the aforementioned severity of the nerve injury, the potential for spontaneous recovery is reduced in more severely injured nerves, which take longer to recover or may not recover at all.

The patient, in this case, suffered avulsion injury to the infraorbital nerve, i.e., neurotmesis according to Sunderland's classification, grade $\mathrm{V}$, during a trauma where the zygomaticomaxillary complex fracture occurred. On CT, comminuted fracture was found in the anterior wall of the maxilla where the infraorbital foramen is located, and avulsion injury was observed in the identified nerve branch due to compression and stretching. As the proximal end of the infraorbital nerve and its branches distributed into other areas was not identified in the vicinity of the infraorbital foramen, it was assumed that the actual injury site was located more proximally. The upper gingival mucosa receives sensory fibers from the trunk of the maxillary nerve as it runs towards the infraorbital canal. In our patient the sensory function of the upper gingiva was intact. From this finding we can assume that the site of nerve damage must have been distal to the maxillary nerve trunk.

Accordingly, it could be assumed that the injury in this case was nerve injury by compression and stretching due to fracture around the infraorbital canal. Although this was confirmed intraoperatively, neurorrhaphy could not be performed as the avulsion occurred in the most proximal area, and conservative treatment and rehabilitation were provided instead.

A sensory nerve conduction study and infraorbital blink reflex test were planned as electrodiagnostic tests to evaluate infraorbital neuropathy. Judging by the notable decrease in amplitude despite a relatively normal sensory nerve action potential latency observed in this patient, the results of the sensory nerve conduction study confirmed axonal death because of trauma. Meanwhile, the gradual increase in amplitude over time suggests the recovery of the infraorbital nerve. After 18 months, the patient showed a $52 \%$ recovery compared to the healthy side.

In the infraorbital blink reflex test, there was a delay or absence in the ipsilateral R1 (early response) and R2 (late response) and the contralateral R2 when stimulating the left side, and a delay in the contralateral R2 when stimulating the right side, which indicates a neuropathy of the left trigeminal nerve, especially the infraorbital nerve, as well as a lesion of the left facial nerve [7]. Furthermore, the blink reflex test showed recovery of the neuropathy since there was a gradual decrease in the latency delay.

Needle electromyography and a motor nerve conduction study were additionally performed to evaluate the facial neuropathy observed in the blink reflex test. Electromyography showed abnormal spontaneous activity and polyphasic motor unit action potentials with a discrete interference pattern, which can be diagnosed as secondary myopathy of the left nasalis and orbicularis oris caused by neuropathy. This demonstrates an injury in the buccal branch of the facial nerve, which innervates the left nasalis and orbicularis oris. When a motor nerve conduction study was performed on nasalis based on these findings, gradual recovery was once again observed over time (Table 1).

The patient's numbness gradually became acceptable, and it was objectively observed from the electrodiagnostic tests that his nerve functions were improving. Hence, assuring and observing a patient that experiences an infraorbital nerve injury due to midfacial fracture and complains of numbness and pain is a plausible option, since even the most severe nerve injury can lead to gradual recovery as in this case, even though microsurgery such as neurorrhaphy or nerve graft was not performed.

Previous studies did not show complete recovery in transected axonal injuries during 1-year follow-ups after microsurgical repair in patients with injury of the trigeminal nerve; further, in cases of trigeminal nerve repair, the subjective and objective sensory recovery was observed in $86 \%$ and $75 \%$ of the patients, respectively, using the two-point discrimination test. Most of the cases showed mild numbness and neuropathic pain even after microsurgical repair. Although a quantitative comparison was not feasible owing to the absence of previous studies on nerve conduction after repair in trigeminal nerve injury, a recovery of $20 \%$ to $64 \%$ was seen in the sensory nerve action potential for a year when sensory nerve conduction was performed without repair. In this study, similarly, a recovery of $37.4 \%$ and $52 \%$ was observed at 12 - and 18-month follow-ups, respectively. Patients who did not undergo surgical repair showed little difference in the recovery pattern; however, a significant difference in the recovery time was seen compared with those undergoing repairs. Sensory dermatome recovery demonstrated different patterns depending on the injury site [8-10].

The mechanism of infraorbital nerve recovery is believed to involve proximal nerve regeneration or compensatory innervation in nearby sensory nerves, such as the zygomatico-facial nerve, external nasal nerve, or buccal nerve. Electrodiagnostic testing results may explain recovery of nerve function, but as reported in literature [11], nerve biopsy or molecular biological 
research is required to determine the exact mechanism.

Additional research on a larger number of patients will be needed to quantify the expected level of recovery objectively, and a continuous follow-up study will be required to examine the limitations of final nerve function recovery following conservative treatment. More data will be needed, but by observing patients for about 18 months, we should be able to educate the patients that can expect a significant improvement in infraorbital nerve function recovery.

\section{NOTES}

\section{Conflict of interest}

No potential conflict of interest relevant to this article was reported.

\section{Ethical approval}

The study was approved by the Institutional Review Board of Chonnam National University Hospital (IRB No. CNUH-2020195) and performed in accordance with the principles of the Declaration of Helsinki. Written informed consent was obtained.

\section{Patient consent}

The patient provided written informed consent for the publication and the use of his images.

\section{ORCID}

Sam Yong Lee https://orcid.org/0000-0002-3185-2519 Seung Hyun Kim https://orcid.org/0000-0001-8269-2036 Jae Ha Hwang https://orcid.org/0000-0001-6992-8067

Kwang Seog Kim https://orcid.org/0000-0002-6766-4640

\section{REFERENCES}

1. Nam Y, Bahk S, Eo S. Anatomical study of the infraorbital nerve and surrounding structures for the surgery of orbital floor fractures. J Craniofac Surg 2017;28:1099-104.

2. Caillaud M, Richard L, Vallat JM, Desmouliere A, Billet F. Peripheral nerve regeneration and intraneural revascularization. Neural Regen Res 2019;14:24-33.

3. Noor M, Ishaq Y, Anwar MA. Frequency of infra-orbital nerve injury after a zygomaticomaxillary complex fracture and its functional recovery after open reduction and internal fixation. Int Surg J 2017;4:685-9.

4. Hong WT, Choi JH, Kim JH, Kim YH, Yang CE, Kim J, et al. Trigeminal somatosensory evoked potential test as an evaluation tool for infraorbital nerve damage. Arch Craniofac Surg 2019;20:223-7.

5. Cho SE, Shin HS, Tak MS, Kang SG, Lee YS, Kim HS, et al. A rare complication of infraorbital nerve hyperesthesia in surgically repaired orbital fracture patients. J Craniofac Surg 2017; 28:e233-4

6. Recknor JB, Mallapragada SK. Nerve regeneration: tissue engineering strategies. In: Bronzino JD, editor. The biomedical engineering handbook. 3rd ed. New York: Taylor and Francis; 2006. p. 48.1-22.

7. Ohki M, Takeuchi N. Objective evaluation of infraorbital nerve involvement in maxillary lesions by means of the blink reflex. Arch Otolaryngol Head Neck Surg 2002;128:952-5.

8. Iro H, Bumm K, Waldfahrer F. Rehabilitation of the trigeminal nerve. GMS Curr Top Otorhinolaryngol Head Neck Surg 2005; 4:Doc12

9. Jaaskelainen SK, Teerijoki-Oksa T, Virtanen A, Tenovuo O, Forssell $\mathrm{H}$. Sensory regeneration following intraoperatively verified trigeminal nerve injury. Neurology 2004;62:1951-7.

10. Krarup C, Archibald SJ, Madison RD. Factors that influence peripheral nerve regeneration: an electrophysiological study of the monkey median nerve. Ann Neurol 2002;51:69-81.

11. Minde J, Svensson O, Toolanen G, Lonne-Rahm SB. Compensatory alterations in dermal innervations in patients with congenital insensitivity to pain. Glob J Rare Dis 2019;4:022-6. 